\title{
Clinical Experience on Umbilical Cord Mesenchymal Stem Cell Treatment in 210 Severe and Critical COVID-19 Cases in Turkey
}

Nesrin Ercelen ( $\nabla$ nesrinercelen@gmail.com )

Department of Medical Genetics, Faculty of Medicine, Haliç University, İstanbul, Turkey https://orcid.org/0000-0002-1053-2305

\section{Kubra Cigdem Pekkoc-Uyanik}

Department of Medical Biology, Faculty of Medicine, Haliç University, İstanbul, Turkey

\section{Nagihan Alpaydin}

Atigen - Cell Technology Center, Trabzon, Turkey

\section{Gokay Rasit Gulay}

Atigen - Cell Technology Center, Trabzon, Turkey

\section{Murat Simsek}

Geneis, Genetic System Solutions, İstanbul, Turkey

\section{Research Article}

Keywords: MSCs, COVID-19, Stem cell therapy, SARS-CoV-2, Cytokine storm, Immunomodulatory effects

Posted Date: June 10th, 2021

DOl: https://doi.org/10.21203/rs.3.rs-590586/v1

License: (1) This work is licensed under a Creative Commons Attribution 4.0 International License. Read Full License

Version of Record: A version of this preprint was published at Stem Cell Reviews and Reports on July 28th, 2021. See the published version at https://doi.org/10.1007/s12015-021-10214-x. 


\section{Abstract}

Objective

Treatment for COVID-19 is still urgent need for the critically ill and severe cases. UC-MSC administration has a therapeutic benefit for severe COVID-19 patients even in the recovery period. In this paper, we aimed to present our clinical experience with UC-MSC treatment in severe and critical severe COVID-19 patients.

Methods

In this study we evaluated the clinical outcome of severe/critically severe 210 COVID-19 patients treated with UC-MSCs, $1-2 \times 10^{6}$ per kilogram to 210 patients from 15/10/2020 until 25/04/2021.

Results

Out of 99 critically severe intubated patients we have observed good clinical progress/discharged from ICU in 52 (52.5\%) patients. Where as 86 (77.5\%) of 111 severe unintubated patients discharged from ICU. Intubated $47(47.5 \%)$ patients and unintubated $25(22.5 \%)$ patients pass away. Significantly higher survival was observed in patients who underwent UC-MSCs before intubation (OR=1.475, 95\% $\mathrm{Cl}=1.193-$ $1.824 \mathrm{p}<0.001$ ). It was observed that the $\mathrm{SaO}_{2}$ parameter tended to improve after UC-MSC therapy compared to all groups. But $\mathrm{SaO}_{2}$ parameter between intubated and unintubated groups was not statistically significant ( $p>0.05$ ), while in discharged cases $\mathrm{SaO}_{2}$ parameter was statistically significant $(p=0.01)$. Besides, there was a statistically significant relation with intubation status, age $(\mathrm{OR}=3.868$, $95 \% \mathrm{Cl}=0.574-7.152 p=0.02)$ and weigh $(\mathrm{OR}=6.768,95 \% \mathrm{Cl}=3.423-10.112 p<0.001)$ thus presented an elevated risk for COVID-19. The linear regression analysis confirmed that the high weight was associated with the risk of intubation in COVID-19 $(p=0.001)$.

Conclusions

According to our results and from recent studies, UC-MSC treatment is safe with high potential to be used as an added therapeutic treatment for severe COVID-19 patients. Our experience showed that UC-MSC therapy may restore oxygenation and downregulate cytokine storm in patients hospitalized with severe COVID-19. We advice wider randomised studies to discover the detailed therapeutic pathophysiology of the MSCs on COVID-19 patients.

\section{Introduction}

COVID-19 causes fever, cough, respiratory tract inflammation, acute respiratory distress syndrome (ARDS), viral sepsis and multisystemic disease in which antigens related to the virus have been detected in most organs [1, 2].

As the virus grew and spread expeditiously in China and around the globe, the World Health Organization (WHO) officially declared COVID-19 a pandemic [3]. Currently, no effective drug alone has been proven to 
cure patients with COVID-19 infection. Thus, the virus is still spreading through population, as of 6 May 2021 , there have been $155,506,494$ confirmed cases and 3,247,228 deaths. [4]. New therapeutic strategies are needed to reduce the death rate and to improve the recovery of patients without side effects. COVID19 triggers exaggerated and constant cytokine production suggesting the virus can stimulate a cytokine storm in the lung, which can also lead to severe organ injury and death [5-7].

The nasal epithelium was discovered to be the principal site of disease and transmission among people with suggestive and asymptomatic SARS-CoV-2 contaminations [8]. Similar to SARS-CoV, the S protein of SARS-CoV-2 ties to angiotensin-converting enzyme receptor 2 (ACE2) and enters cells in a way catalyzed by transmembrane protease serine 2 (TMPRSS2) in type II alveolar epithelial cells $[8,9]$. The $S$ protein of SARS-CoV-2 for ACE2 is the fundamental determinant of the replication rate of SARS-CoV-2 [10]. Regardless of critical advances in supportive treatment strategies, the incidence and mortality of ARDS stay high [11].

Stem cells derived from different tissues, such as human bone marrow, adipose tissue, and umbilical cord tissue (UC-MSC) have been widely used for regulating immunomodulation by their paracrine effects with different types of cytokines in autoimmune diseases [12]. According to the clinical trials in COVID-19 patients, with UC-MSC and placebo group; an improvement in pulmonary involvement and a better recovery have been demostrated in patients with UC-MSC injection [13]. This finding indicates that UCMSC administration has a therapeutic benefit for severe COVID-19 patients even in the recovery period [13-15]. In this paper, we aimed to present our clinical experience with UC-MSC treatment in severe and critical severe 210 COVID-19 patients.

\section{Materials And Methods}

\section{Experimental design}

Intravenous UC-MSCs transplantation was performed on 210 patients followed in ICU and COVID wards. These 210 patients were clinically heterogeneous and unresponsive to unique COVID-19 medical treatment algorithms confirmed with Turkish Ministry of Health (advised clinical treatment). The patients were enrolled into the supportive treatment with each family's signed written consent form, in accordance with the Declaration of Helsinki. The UC-MSC transplantations were performed in several hospitals in Turkey and approved by ministry of health stem cell commission (COVID-19) online registration system in 24 hours.

\section{Cell Preparation And Transplantation}

The clinical grade UC-MSCs were supplied by the Atigen-Cell Technology Center, Trabzon, Turkey licensed by Turkish Health Ministry. The total number of cell count used was $1-2 \times 10^{6}$ cells per kilogram. The cells were prepared for injection in $100 \mathrm{ml}$ of normal saline. The UC-MSCs were administered to the patients at a critical stage, when they did not show any improvement by the recommended clinical treatment for COVID-19, as to whether the effectiveness of the cellular treatment on this severe stage of 
infection and inflammation could be observed. The cells were infused in 50-60 minutes with a rate of 2 $\mathrm{ml} /$ minute, as described in the literature $[5,15)$. In this study we evaluated the outcome of the MSC treatment after a single dose of clinical-grade UC-MSCs $\left(1-2 \times 10^{6}\right.$ cells $\left./ \mathrm{kg}\right)$, intravenously.

\section{Patients}

The patients (ages 23-81) were tested by the real-time Reverse Transcription Polymerase Chain Reaction (RT-PCR) assay of nasal and pharyngeal swabs and evaluated when they were first admitted. In case of one of them confirmed with COVID-19 infection, the patient was started with the recommended treatment protocol by the ministry of health, and a second PCR test was scheduled to hospital. Patients were excluded from the study if they had a history of cancer or bacterial and yeast secondary infection. The patients were enrolled for the supportive UC-MSC transplantation due to their lack of response to advised treatments. Patients were followed in intensive care unit (ICU) and COVID wards for primary safety and efficacy outcomes as introduced in previous COVID-19 studies in the literature, until they were discharged from the hospital or passed away. All the patients were underwent MSCs transplantation after the approval by Stem Cell Treatment Comission of Turkish Health Ministry. This procedure is an active online protocol between the hospital clinic and Ministry Commission; followed by clinical doctor's signed requisation form, patient representative signed consent and Licenced Stem Cell Center cell product signed form.

The cells are carried to the hospitals under the confirmed "transport protocol" of the Cell Center quality system. Intravenous MSC transplantation was performed on 210 PCR positive severe and critically severe COVID-19 patients followed in inpatient/ICU, unresponsive to treatment algorithms advised by the Turkish COVID-19 Scientific Committee. As the 17 patients excluded from the study, they had a history of cancer or bacterial and yeast secondary infection.

\section{Statistical analysis}

The findings of this study were evaluated by using the software IBM SPSS Statistics (IBM Corporation version 20.0 SPSS Inc., Chicago, IL, USA). Quantitative data were shown as mean ( \pm standard deviation), whereas categorical data were shown as numbers and percentiles. When comparing normally distributed continuous variables between the study groups, $t$-test was used. Qualitative data such as sex, intubated condition, outcome condition, safety and efficiency outcome were tested by using the Chi-square statistic. We evaluated the comparision of arterial oxygen saturation $\left(\mathrm{SaO}_{2}\right)$ before and after UC-MSC therapy by Paired Sample $t$-test. A multivariate analysis was performed by the linear regression model. The model included age and weight as independent variables and intubation condition was used as a dependent variable. To determine the risk factor between groups, odds ratio (OR) and $95 \%$ confidence interval (95\% $\mathrm{Cl})$ were used. A $p$-value of $<0.05$ was regarded statistically significant.

\section{Results}




\section{Participant flow}

COVID-19 infected critically severe and severe patients were evaluated for this study. Participant flow chart with enrollment and randomization is shown in Fig. 1. They were followed with unique COVID-19 medical treatment algorithms confirmed by Turkish Ministry of Health (advised clinical treatment). Every patient received UC-MSCs (1-2 $\times 10^{6}$ per kilogram) on average 6.4 days after being tested positive. Demographics and baseline characteristics for enrolled patients are presented in Table 1. 99 critically severe patients were intubated before the MSC transplantation. All the patients were observed until they were discharged from ICU or passed away.

Table 1

Baseline characterictics of treatment group

UC-MSC

\begin{tabular}{|c|c|c|c|c|}
\hline Characteristics & Intubated $(n=99)$ & Unintubated $(n=111)$ & Total (n) & $P$ value \\
\hline Sex, n (\%) & & & 210 & $0,64^{a}$ \\
\hline Male & $74(74.7 \%)$ & $79(71.2 \%)$ & 153 & \\
\hline Female & 25 (25.3\%) & $32(28.8 \%)$ & 57 & \\
\hline Age, mean $\pm S D$, years & $61.22 \pm 11.77$ & $57.36 \pm 12.00$ & & $0,02^{b}$ \\
\hline Age range, years & $27-83$ & $23-81$ & & \\
\hline Weight, mean $\pm \mathrm{SD}, \mathrm{kg}$ & $90.63 \pm 12.86$ & $83.86 \pm 11.66$ & & $<0,001^{b}$ \\
\hline \multicolumn{5}{|c|}{ Abbreviations: UC-MSC: umbilicial cord mesenchymal stem cell. n: number of samples. } \\
\hline \multicolumn{5}{|c|}{ a Pearson Chi-square test. ${ }^{b} t$-test. } \\
\hline \multicolumn{5}{|c|}{$\begin{array}{l}\text { In this study, patients who were intubated were in the critical disease category, while patients who } \\
\text { were not intubated were in the severe disease category. COVID- } 19 \text { disease severity; Mild, moderate, } \\
\text { severe and critically disease. Severe Illness: Individuals who have SpO } 2<94 \% \text { on room air at sea level } \\
\text { a ratio of arterial partial pressure of oxygen to fraction of inspired oxygen (PaO2/FiO2) }<300 \mathrm{~mm} \mathrm{Hg} \text {, } \\
\text { respiratory frequency }>30 \text { breaths/min, or lung infiltrates }>50 \% \text {. Critical Illness: Individuals who have } \\
\text { respiratory failure, septic shock, and/or multiple organ dysfunction (3) }\end{array}$} \\
\hline
\end{tabular}

\section{Recruitment}

Patients were excluded from the study if they had a history of cancer or bacterial and yeast secondary infection, so excluded 17 out of 227 patients. 210 severe and critically severe patients recieved UC-MSCs. UC-MSCs derived from Wharton's jelly tissue were used in Turkey on 210 severe/critically ill COVID-19 patients, $47.1 \%$ of the patients were intubated in the ICU and $52,9 \%$ of the patients were unintubated.

\section{Primary Safety Outcome}


No adverse effects were observed related to infusion or allergic reactions, secondary infection, or lifethreatening adverse events in patients, who received UC-MSC transplantation. The treatments were recorded within the predicted safety levels of UC-MSC transplantation treatments mentioned in the previous studies.

\section{Efficacy Outcome}

We evaluated patients in two main groups, the first group involved the patients who were unintubated. The second group involved the intubated patients. Patients in both groups were followed-up for at least 2-3 weeks after UC-MSC injection. We evaluated the patient exit with the clinical condition and comparision of $\mathrm{SaO}_{2}$ contributing to the efficacy outcome after UC-MSC injection.

A total of 210 cases diagnosed with COVID-19 were studied. 57 patients were female, and 153 patients were male with mean ages of $56.84 \pm 14.12$ and $59.99 \pm 11.06$, respectively. There was not statistically significant difference between men and women in the terms of age $(p=0.09)$. While the mean age of intubated patients is $61.22 \pm 11.77$, the mean age of unintubated patients is $57.36 \pm 12.00$. There was a statistically significant relation between intubation status in terms of age $(\mathrm{OR}=3.868,95 \% \mathrm{Cl}=0.574-$ $7.152 p=0.02$ ). High age is associated with the risk of intubation in COVID-19. The mean weight of intubated patients is $90.63 \pm 12.86$, while the mean weigh of unintubated patients is $83.86 \pm 11.66$. There was a statistically significant relation between intubation status in terms of weigh $(\mathrm{OR}=6.768,95 \% \mathrm{Cl}=$ 3.423-10.112 $\mathrm{p}<0.001$ ) (Table 1). High weight is associated with the risk of intubation in COVID-19.

It was observed that the $\mathrm{SaO}_{2}$ parameter tended to improve after UC-MSC therapy compared to all groups. But $\mathrm{SaO}_{2}$ parameter between intubated and unintubated groups was not statistically significant $(p>0.05)$, while in discharged cases $\mathrm{SaO}_{2}$ parameter was statistically significant $(p=0.01)$ (Table 2$)$.

Table 2

Comparision of $\mathrm{SaO}_{2}$ before and after UC-MSC therapy

\begin{tabular}{|llll|}
\hline $\mathrm{SaO}_{2}$ & Before UC-MSC therapy & After UC-MSC therapy & $\boldsymbol{P v a l u e}^{\mathrm{a}}$ \\
\hline Intubated & $90.05 \pm 6.49$ & $90.34 \pm 5.95$ & 0.35 \\
\hline Unintubated & $92.47 \pm 4.52$ & $92.76 \pm 4.52$ & 0.16 \\
\hline Discharged & $92.09 \pm 5.47$ & $92.63 \pm 4.81$ & $\mathbf{0 . 0 1 *}$ \\
\hline Total & $91.34 \pm 5.64$ & $91.63 \pm 5.40$ & 0.11 \\
\hline Abbreviations: $\mathrm{SaO}_{2}$ : Arterial oxygen saturation. UC-MSC: umbilicial cord mesenchymal stem cell. \\
\hline \multicolumn{2}{|l}{ Paired Sample $t$ Test. Values are included as mean \pm standard deviation. } \\
\hline
\end{tabular}

Out of 99 critically severe intubated patients we have observed good clinical progress/discharged from ICU in 52 (52.5\%) patients. Where as 86 (77.5\%) of 111 severe unintubated patients discharged from ICU. 
Intubated $47(47.5 \%)$ patients and unintubated 25 (22.5\%) patients pass away. Significantly higher survival was observed in patients who underwent UC-MSCs before intubation $(\mathrm{OR}=1.475,95 \% \mathrm{Cl}=$ 1.193-1.824 $p<0.001$ ) (Table 3). Besides, exit status in COVID-19 patients treated with MSCs according to intubation status was shown that Fig. 2.

Table 3

Outcomes of patients who underwent UC-MSC according to the intubation condition

\begin{tabular}{|c|c|c|c|c|}
\hline $\begin{array}{l}\text { Intubated } \\
\text { condition }\end{array}$ & $\begin{array}{l}\text { Discharged / Treatment Continues n } \\
\text { (\%) }\end{array}$ & $\begin{array}{l}\text { Death n } \\
\text { (\%) }\end{array}$ & Total n (\%) & $\begin{array}{l}P \\
\text { value }^{a}\end{array}$ \\
\hline & & & & $<0.001 *$ \\
\hline Intubated & $52(52.5 \%)$ & $47(47.5 \%)$ & $99(100 \%)$ & \\
\hline Unintubated & $86(77.5 \%)$ & $25(22.5 \%)$ & $\begin{array}{l}111 \\
(100 \%)\end{array}$ & \\
\hline Total & $138(61 \%)$ & $72(39.0 \%)$ & $\begin{array}{l}210 \\
(100 \%)\end{array}$ & \\
\hline \multicolumn{5}{|c|}{ Abbreviations: $\mathrm{n}$ : number of samples. UC-MSC: umbilicial cord mesenchymal stem cell. } \\
\hline \multicolumn{5}{|c|}{ a Pearson Chi-square test. Values are given as the number of subjects ( $n$ ) and percentages (\%). } \\
\hline \multicolumn{5}{|c|}{$*(\mathrm{OR}=1.475,95 \% \mathrm{Cl}=1.193-1.824 p<0.001)}$. \\
\hline
\end{tabular}

In the linear regression model, the dependent variable, intubation condition was significantly associated with weight $(p=0.001)$. The linear regression analysis confirmed that the high weight was associated with the risk of intubation in COVID-19 (Table 4).

Table 4

Linear regression analysis of risk factors for the COVID-19

\begin{tabular}{|llllll|}
\hline Independent variables & B & Std. Error & Standardized Beta & P value & 95\% Cl for B \\
\hline Years & 0.004 & 0.003 & 0.089 & 0.208 & $(-) 0.002-(-) 0.009$ \\
\hline Weight & 0.010 & 0.003 & 0.247 & 0.001 & $0.004-0.015$ \\
\hline \multicolumn{7}{l}{ Dependent variable: Intubated condition. } & & & \\
\hline
\end{tabular}

\section{Discussion}

COVID-19 causes fever, cough, respiratory tract inflammation, acute respiratory distress syndrome (ARDS), viral sepsis and multisystemic disease [1, 2]. Clinical experience thus far shows that COVID-19 is highly heterogeneous, ranging from being asymptomatic and mild to severe and causing death. Host factors including age, sex, and comorbid conditions are key determinants of disease severity and progression. Aging itself is a prominent risk factor for severe disease and death from COVID-19 [16]. In our study, A total of 210 cases diagnosed with COVID-19 were studied. 57 patients were female, and 153 
patients were male with mean ages of $56.84 \pm 14.12$ and $59.99 \pm 11.06$, respectively. There was not statistically significant difference between men and women in the terms of age $(p=0.09)$. While the mean age of intubated patients was $61.22 \pm 11.77$, the mean age of unintubated patients was $57.36 \pm 12.00$. There was a statistically significant relation between intubation status in terms of age $(\mathrm{OR}=3.868,95 \%$ $\mathrm{Cl}=0.574-7.152 p=0.02)$.

Popkin et al. shown in meta-analysis that individuals with obesity were more at risk for COVID-19 positive, $>46.0 \%$ higher $(\mathrm{OR}=1.46 ; 95 \% \mathrm{Cl}, 1.30-1.65 ; \mathrm{p}<0.0001)$; for hospitalization, $113 \%$ higher $(\mathrm{OR}=2.13 ; 95 \%$ $\mathrm{Cl}, 1.74-2.60 ; \mathrm{p}<0.0001)$; for ICU admission, $74 \%$ higher $(\mathrm{OR}=1.74 ; 95 \% \mathrm{Cl}, 1.46-2.08)$; and for mortality, $48 \%$ increase in deaths $(\mathrm{OR}=1.48 ; 95 \% \mathrm{Cl}, 1.22-1.80 ; p<0.001)$ [17]. In our study, while the mean weight of intubated patients was $90.63 \pm 12.86$, the mean weigh of unintubated patients was $83.86 \pm 11.66$. There was a statistically significant relation between intubation status in terms of weigh (OR $=6.768,95 \%$ $\mathrm{Cl}=3.423-10.112 \mathrm{p}<0.001)$. In the linear regression model, the dependent variable, intubation condition was significantly associated with weight $(p=0.001)$. The linear regression analysis confirmed that the high weight was associated with the risk of intubation in COVID-19.

Mesenchymal stem cells are considered to have extensive clinical application possibilities, including ARDS [18]. MSCs have broad bioactivities, including repair, immunomodulation, increased alveolar fluid clearance, and regulation of pulmonary vascular endothelial permeability [19]. MSCs have a wide range of sources, such as the bone marrow, umbilical cord, adipose tissue, amniotic membrane, and several other tissues [20]. MSCs from different sources have significant similarities, such as being adherent and teardrop- or spindle-shaped [21]. MSCs have the upsides of self-renewal, multidirectional differentiation, and immunomodulation towards suppressing proinflammatory factors. In Accord with these findings, it has been shown that stem cells from the placenta and human umbilical cord showed decreased lung tissue damage in mouse bleomycin models [22]. In clinical setting, immunomodulatory properties of MSCs have been shown in numerous pathological conditions, e.g., bronchopulmonary dysplasia, asthma, acute lung injury, chronic obstructive pneumonic illness, idiopathic aspiratory fibrosis [23, 24]. Studies suggest that MSCs from different sources have different levels of immunoregulatory efficiency. Najar and his colleques revealed in their study that Warton Jelly derived MSCs were found to be the most effective in immunomodulation [25].

Toll-like receptors (TLRs) family has a crucial activity in the innate immune system for the recognition of pathogen-associated molecular patterns (PAMPs), starting primary reaction to pathogens and helping to recognise virus, bacteria, protozoa, and fungi; and are commonly associated with chronic inflammatory and autoimmune diseases [26]. TLRs play an important role in immunomodulation by cell-to-cell contact and MSC-secreted soluble factors $[27,28]$.

It has been reported that some patients who survived acute phase of ARDS due to COVID-19 die afterwards due to progressive pulmonary fibrosis [29]. Disproportionate activation and proliferation of myofibroblasts results in deposition of the extracellular matrix (ECM) components which deteriorates functions of related tissues and fibrotic states are estimated to contribute to almost $50 \%$ of mortalities in 
the developed world [30]. During inflammatory phase of ARDS, dysregulation and overproduction of matrix metalloproteinases could occur and lead to a complex combination of epithelial and endothelial damage, thus uncontrolled fibrosis [31].

Overactive secretion of pro-fibrotic growth factors, chemokines and procoagulant mediators are collectively referred as senescence-associated secretory phenotype (SASP) factors. Secretion of these mediators trigger progressive and excessive activation of epithelial cells characterized by dysregulated crosstalk between epithelial cells and mesenchymal cells. Consequent accumulation and activation of myofibroblasts affect the crosstalk between fibroblasts and epithelial cells to exhibit markers of stress and senescence which in turn lead to resistance to apoptosis and excessive production of extracellular matrix components $[32,33]$.

Inflammation in lung tissue results in alveolar pneumocyte damage and resultant fibroblast activation triggers pulmonary fibrosis through myofibroblast differentiation which might proceed for a long time [34]. Darwish et al. have shown that umbilical cord derived MSCs (UC-MSCs) have the potential to alleviate H5N1 infection induced acute lung injury. Inflammatory cytokine profiles seen in H5N1 infection is similar to COVID-19 such as high levels of IL-6, GCSF, IP10, MCP-1, MIP1 a, and TNF-a [6, 35, 36]. These inflammatory cytokines can activate MSCs to secrete immunosuppressive factors consisting of IDO, TSG6, NO, IL-10, CCL2, galectins, PGE2, and TGF- $\beta$ and then modulating tissue homeostasis [37].

In recent studies clinical improvement was observed in severe COVID-19 patients, shortening the ICU stay $[13,14]$. In a proof-of-concept study significant benefit of laboratory prognostic markers was evaluated consistent with other study findings in COVID-19 patients who had received MSCs transplantation [15, 38]. In another clinical trial with randomised, double-blind, placebo-controlled of phase 2 were done on 100 severe COVID-19 patients with lung damage. They were randomly assigned to receive either UCMSCs $4 \times 107$ cells per infusion to 65 patients or placebo (35 patients) on day 0,3 , and 6 . It was shown that UC-MSCs treatment is safe and have promissing reatment potential for COVID-19 patients. Improvement in whole lung lesion volume were reported with a better recovery rate compared to the placebo group [13].

Important pathological hallmark of COVID-19 in pulmonary vasculature is pulmonary hypertension as a part of systemic endothelial dysfunction [39, 40]. Endothelial dysfunction and damage explain most of the pathological processes triggered and involved in COVID-19 and mesenchymal cells seem to be promising agents to alleviate such widespread cellular damage [41, 42].

Despite ever increasing number of studies, randomized controlled multicenter clinical trials are certainly needed to achieve standardization of MSC therapy protocols for cell-based treatments in COVID-19 [5, 15, 43-45].

This study was investigated the impact of UC-MSCs therapy in 210 patients with COVID-19 (99 patient with critically severe, 111 patients with severe clinical symptoms). Patients were followed after by pulmonary function and clinical symptoms after the injection. Patients were observed for 2-3 weeks 
after MSCs transplantation. In our study we have revealed a clinical success rate of $61 \%$ in UC-MSC transplanted severe/critically severe COVID-19 patients. We have also observed that the response to MSC treatment is more accurate in severe patients during early stages of cytokine storm than the patients who were entubated and had lung incury due to the hyperimmunity tissue destruction. The results showed that significantly higher survival was observed in patients who underwent UC-MSCs before intubation $(p<$ $0.001)$.

Here we could discuss about the two phases of UC-MSC treatment in COVID-19 disease due to the clinical aspects we have observed in these patients (Fig. 3). In this study, severe patients who were unintubated were in the Phase I, while critical patients who were intubated were in the Phase II. In Phase I, we assume to alleviate the cytokine storm in severe COVID-19 patients with MSC transplantation. The immunmodulatory efficacy of MSCs has been revealed in many studies $[7,12,46]$. In this period of the disease, we could conclude to achieve a beneficial result of the UC-MSC treatment by a proven pathway of immun regulation. We could recommend one iv MSCs dose in the literature as $1-2 \times 10^{6} / \mathrm{kg}$ as effective for phase I COVID-19 patients [46]. Phase II (supportive treatment for organ recovery): Here we could comment on the treatment mechanism of the MSCs as a supportive treatment for original healing of the alveolar epithelial cells in critically severe COVID-19 patients. This may be achieved by anti inflammatory/antifibrosis supportive effect of MSCs which was determined in various studies $[5,6,35$, 36]. Anti inflammatory supportive effect provides to increase oxygenation in destroyed tissue, which gaines time for patient organ recovery. In our study, it was observed that the $\mathrm{SaO}_{2}$ parameter tended to improve after UC-MSC therapy compared to all groups. Especially, in discharged cases $\mathrm{SaO}_{2}$ parameter was statistically significant $(p=0.01)$.

UC-MSCs supportive treatment as prevention of fibrosis, which is seen in the COVID-19 patients as a disease complication is vital. We have scientific evidence that the accelerated regenerative original tissue healing may be achieved with MSCs treatment, but this may take some time not less than a couple of weeks, which was explored in many studies [47].

\section{Conclusion}

In the first stage of COVID-19 disease, alleviate UC-MSC transplantation is concluded as effective to alleviate the cytokine storm by immune regulation which leads improvement in patient clinical outcome. In the second stage of the disease, MSCs transplantation is evaluated as to increase oxygenation in mostly undamaged lung tissue by an anti-inflammatory effect. However, this effect does not occur constantly. In this condition we may advice repeated multiple doses supporting patient treatment.

In intubated COVID-19 patients with lung or organ injuries we may possibly support the original tissue healing by MSCs transplantation in long term follow up, but the dose and clinical application protocol is much different from the treatment protocol than we use in phase I. We could recommend single iv infussion with $1-2 \times 10^{6}$ cells $/ \mathrm{kg}$ in phase I. Thus, we have observed that repeated doses may be recomended in phase II patients with tisuue injury for an ongoing healing support by mostly anti- 
inflammatory and immunregulatory effect. The dose and the application intervals could be experienced by each patient's clinical response to the treatments.

Immunomodulatory/anti-infamatory and regenerative properties of UC-MSCs have demonstrated promising outcomes on numerous pulmonary disease states. Administration of UC-MSCs to COVID-19 patients has been shown to be well tolerated and safe without any detectable side effect. In the literature so far even patients with severe COVID-19 and pulmonary inflammation have demonstrated better rates of clinical outcome. UC-MSCs have the potential to alleviate inflammation and cytokine storm in COVID19 patients, together with reducing pulmonary fibrosis processes. Still randomized clinical trials are needed to assess the exact mechanisms of action to determine the best route of application/dosage to enhance short- and long-term UC-MSCs therapeutic outcome.

We advise urgent randomised multicenter studies to evaluate the clinical experience achieved in our clinics and to discuss the mechanisms for the MSCs teatment strategies which we could have revealed some basic facts of the literature related by MSCs treatments.

\section{Abbreviations}

COVID-19

Coronavirus Disease 2019

MSCs

Mesenchymal stem cells

UC-MSCs

Umbilical Cord-derived mesenchymal stem cells

SARS-CoV-2

Severe Acute Respiratory Syndrome Coronavirus 2

ARDS

Acute respiratory distress syndrome

WHO

World Health Organization

TMPRSS2

Transmembrane Serine Protease 2

RT-PCR

Real Time PCR

ICU

Intensive care unit

TLRs

Toll-like receptors

PAMPs

Pathogen-associated molecular patterns

ECM 
The extracellular matrix

SASP

Senescence-associated secretory phenotype

IL-6

Interleukin 6

GCSF

Granulocyte colony-stimulating factor

IP10

Interferon gamma-induced protein 10

MCP-1

The monocyte chemoattractant protein-1

MIP1a

Macrophage inflammatory protein $1 a$

TNF-a

Tumor necrosis factor-alpha

\section{Declarations}

Author information

\section{Affiliations}

Department of Medical Genetics, Faculty of Medicine, Haliç University, İstanbul, Turkey

Nesrin O. Ercelen

Department of Medical Biology, Faculty of Medicine, Haliç University, İstanbul, Turkey

Kubra Cigdem Pekkoc-Uyanik

Atigen - Cell Technology Center, Trabzon, Turkey

Nagihan Alpaydin

Atigen - Cell Technology Center, Trabzon, Turkey

Gokay Rasit Gulay

Geneis, Genetic System Solutions, İstanbul, Turkey

Murat Simsek

KÇPU 
Methodology, Investigation, Writing- original draft, Conceptualization, Data curation, Writingreview\&editing.

\section{NA \\ Investigation, Validation. \\ GRG \\ Investigation, Validation. \\ MS \\ Investigation, Validation. \\ Consent for Publication}

Not applicable.

\section{Conflict of interest:}

The authors declare that they have no conflict of interests.

\section{Funding}

The present study was not supported by a grant.

\section{Contributions}

NEO: Project administration, Methodology, Writing original draft, Conceptualization, Data curation, Writing-review\&editing.

\section{References}

1. Li H, Liu L, Zhang D, et al. (2020). SARS-CoV-2 and viral sepsis: observations and hypotheses. Lancet. 395(10235),1517-1520.

2. Odabasi Z, Cinel I. (2020). Consideration of Severe Coronavirus Disease 2019 As Viral Sepsis and Potential Use of Immune Checkpoint Inhibitors. Crit Care Explor. 2(6), e0141.

3. WHO characterizes COVID-19 as a pandemic. https://www.who.int/emergencies/diseases/novelcoronavirus-2019/events-as-they-happen. Accessed 05.05.2021

4. WHO Coronavirus (COVID-19) Dashboard, https://covid19.who.int/ (2021). Accessed 05.05.2021. 
5. Leng Z, Zhu R, Hou W, et al. (2020). Transplantation of ACE2- Mesenchymal Stem Cells Improves the Outcome of Patients with COVID-19 Pneumonia. Aging Dis. 11(2),216-228.

6. Huang C, Wang Y, Li X, et al. 2020. Clinical features of patients infected with 2019 novel coronavirus in Wuhan, China [published correction appears in Lancet. 2020 Jan 30;:]. Lancet. 395(10223), 497506.

7. Mehta P, McAuley DF, Brown M, et al. 2020. COVID-19: consider cytokine storm syndromes and immunosuppression. Lancet. 395(10229), 1033-1034.

8. Sungnak W, Huang N, Bécavin C, et al. 2020. SARS-CoV-2 entry factors are highly expressed in nasal epithelial cells together with innate immune genes. Nat Med. 26(5), 681-687.

9. Matsuyama S, Nagata N, Shirato K, Kawase M, Takeda M, Taguchi F. 2010. Efficient activation of the severe acute respiratory syndrome coronavirus spike protein by the transmembrane protease TMPRSS2. J Virol. 84(24), 12658-12664.

10. Hoffmann M, Kleine-Weber H, Schroeder S, et al. 2020. SARS-CoV-2 Cell Entry Depends on ACE2 and TMPRSS2 and Is Blocked by a Clinically Proven Protease Inhibitor. Cell. 181(2), 271-280.e8.

11. Rothlin RP, Vetulli HM, Duarte M, Pelorosso FG. 2020. Telmisartan as tentative angiotensin receptor blocker therapeutic for COVID-19. Drug Dev Res. 81(7), 768-770.

12. Zhou C, Yang B, Tian Y, et al. 2011. Immunomodulatory effect of human umbilical cord Wharton's jelly-derived mesenchymal stem cells on lymphocytes. Cell Immunol. 272(1), 33-38.

13. Shi L, Huang H, Lu X, et al. 2021. Effect of human umbilical cord-derived mesenchymal stem cells on lung damage in severe COVID-19 patients: a randomized, double-blind, placebo-controlled phase 2 trial. Signal Transduct Target Ther. 6(1), 58.

14. Meng F, Xu R, Wang S, et al. 2020. Human umbilical cord-derived mesenchymal stem cell therapy in patients with COVID-19: a phase 1 clinical trial. Signal Transduct Target Ther. 5(1), 172.

15. Ercelen NO, Bilgili B, Monteleone B, et al. 2020. MSC Transplantation in Eight Severe COVID-19 Patients: Can Cytokine Storm Be Reversed? J Stem Cell Res Ther. 10, 460.

16. Chen Y, Klein SL, Garibaldi BT, et al. 2021. Aging in COVID-19: Vulnerability, immunity and intervention. Ageing Res Rev. 65, 101205.

17. Popkin BM, Du S, Green WD, et al. 2020. Individuals with obesity and COVID-19: A global perspective on the epidemiology and biological relationships. Obes Rev. 21(11), e13128.

18. Hashemian SR, Aliannejad R, Zarrabi M, et al. 2021. Mesenchymal stem cells derived from perinatal tissues for treatment of critically ill COVID-19-induced ARDS patients: a case series. Stem Cell Res Ther. 12(1), 91.

19. Walter J, Ware LB, Matthay MA. 2014. Mesenchymal stem cells: mechanisms of potential therapeutic benefit in ARDS and sepsis. Lancet Respir Med. 2(12), 1016-26.

20. Main H, Munsie M, O'Connor MD. 2014. Managing the potential and pitfalls during clinical translation of emerging stem cell therapies. Clin Trans/ Med. 3, 10. 
21. Javazon EH, Colter DC, Schwarz EJ, Prockop DJ. 2001. Rat marrow stromal cells are more sensitive to plating density and expand more rapidly from single-cell-derived colonies than human marrow stromal cells. Stem Cells. 19(3), 219-225.

22. Yang J, Jia Z. 2014. Cell-based therapy in lung regenerative medicine. Regen Med Res. 2(1), 7.

23. Behnke J, Kremer S, Shahzad T, et al. 2020. MSC Based Therapies-New Perspectives for the Injured Lung. J Clin Med. 9(3), 682.

24. Weiss DJ, Bertoncello I, Borok Z, et al. 2011. Stem cells and cell therapies in lung biology and lung diseases. Proc Am Thorac Soc. 8(3), 223-272.

25. Najar M, Raicevic G, Fayyad-Kazan H, et al. 2012. Immune-related antigens, surface molecules and regulatory factors in human-derived mesenchymal stromal cells: the expression and impact of inflammatory priming [published correction appears in Stem Cell Rev. 2012 Dec;8(4):1286. Kazan, Hussein Fayyad [corrected to Fayyad-Kazan, Hussein]]. Stem Cell Rev Rep. 8(4), 1188-1198.

26. Lotfinejad P, Asadzadeh Z, Najjary S, et al. 2020. COVID-19 Infection: Concise Review Based on the Immunological Perspective [published online ahead of print, 2020 Sep 28]. Immunol Invest. 1-20.

27. Pevsner-Fischer M, Morad V, Cohen-Sfady M, et al. 2007.Toll-like receptors and their ligands control mesenchymal stem cell functions. Blood. 109(4), 1422-1432.

28. Lombardo E, DelaRosa O, Mancheño-Corvo P, et al. 2009. Toll-like receptor-mediated signaling in human adipose-derived stem cells: implications for immunogenicity and immunosuppressive potential. Tissue Eng Part A. 15(7), 1579-1589.

29. George PM, Wells AU, Jenkins RG. 2020. Pulmonary fibrosis and COVID-19: the potential role for antifibrotic therapy. Lancet Respir Med. 8(8), 807-815.

30. Friedman SL, Sheppard D, Duffield JS, Violette S. 2013.Therapy for fibrotic diseases: nearing the starting line. Sci Trans/ Med. 5(167), 167sr1.

31. Zemans RL, Colgan SP, Downey GP. 2009. Transepithelial migration of neutrophils: mechanisms and implications for acute lung injury. Am J Respir Cell Mol Biol. 40(5), 519-535.

32. Lopes-Paciencia S, Saint-Germain E, Rowell MC, et al. 2019. The senescence-associated secretory phenotype and its regulation. Cytokine. 117, 15-22.

33. Sgalla G, lovene B, Calvello M, et al. 2018. Idiopathic pulmonary fibrosis: pathogenesis and management. Respir Res. 19(1), 32.

34. Zuo W, Zhao X, Chen YG. 2009. SARS Coronavirus and Lung Fibrosis. Molecular Biology of the SARSCoronavirus. 22,247-58.

35. Darwish I, Mubareka S, Liles WC. 2011. Immunomodulatory therapy for severe influenza. Expert Rev Anti Infect Ther. 9(7), 807-822.

36. Wang D, Hu B, Hu C, et al. 2020. Clinical Characteristics of 138 Hospitalized Patients With 2019 Novel Coronavirus-Infected Pneumonia in Wuhan, China [published correction appears in JAMA. 2021 Mar 16;325(11):1113]. JAMA. 323(11), 1061-1069. 
37. Wang M, Yuan Q, Xie L. 2018. Mesenchymal Stem Cell-Based Immunomodulation: Properties and Clinical Application. Stem Cells Int. 2018, 3057624. Published 2018 Jun 14.

38. Liang B, Chen J, Li T, et al. 2020. Clinical remission of a critically ill COVID-19 patient treated by human umbilical cord mesenchymal stem cells: A case report. Medicine (Baltimore). 99(31), e21429.

39. Yamaoka-Tojo M. 2020. Vascular Endothelial Glycocalyx Damage in COVID-19. Int J Mol Sci. 21(24), 9712.

40. Varga Z. 2020. Endotheliitis bei COVID-19 [Endotheliitis in COVID-19]. Pathologe. 41(Suppl 2), 99102.

41. Zhang S, Liu X, Ge LL, et al. 2020. Mesenchymal stromal cell-derived exosomes improve pulmonary hypertension through inhibition of pulmonary vascular remodeling. Respir Res. 21(1), 71.

42. Fukumitsu M, Suzuki K. 2019. Mesenchymal stem/stromal cell therapy for pulmonary arterial hypertension: Comprehensive review of preclinical studies. J Cardiol. 74(4), 304-312.

43. Sánchez-Guijo F, García-Arranz M, López-Parra M, et al. 2020. Adipose-derived mesenchymal stromal cells for the treatment of patients with severe SARS-CoV-2 pneumonia requiring mechanical ventilation. A proof of concept study. EClinicalMedicine. 25, 100454.

44. Moll G, Drzeniek N, Kamhieh-Milz J, et al. 2020. MSC Therapies for COVID-19: Importance of Patient Coagulopathy, Thromboprophylaxis, Cell Product Quality and Mode of Delivery for Treatment Safety and Efficacy. Front Immunol. 11, 1091.

45. Qu W, Wang Z, Hare JM, et al. 2020. Cell-based therapy to reduce mortality from COVID-19: Systematic review and meta-analysis of human studies on acute respiratory distress syndrome. Stem Cells Trans/ Med. 9(9), 1007-1022.

46. Yadav P, Vats R, Bano A, Bhardwaj R. 2020. Mesenchymal stem cell immunomodulation and regeneration therapeutics as an ameliorative approach for COVID-19 pandemics. Life Sci. 263, 118588.

47. Basiri A, Pazhouhnia Z, Beheshtizadeh N, 2021. Regenerative Medicine in COVID-19 Treatment: Real Opportunities and Range of Promises. Stem Cell Rev Rep. 17(1), 163-175.

\section{Figures}




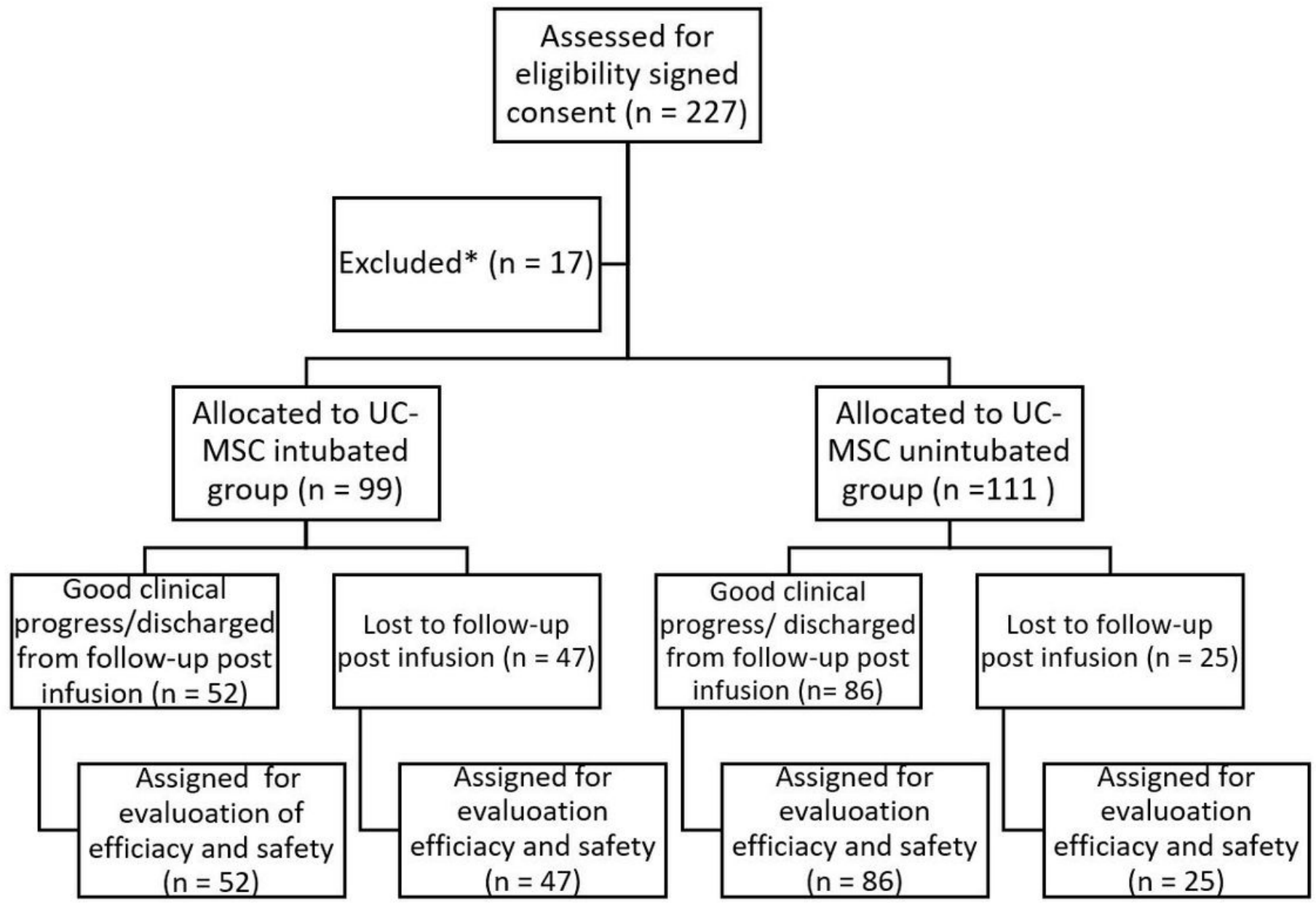

Figure 1

Participant flow. 


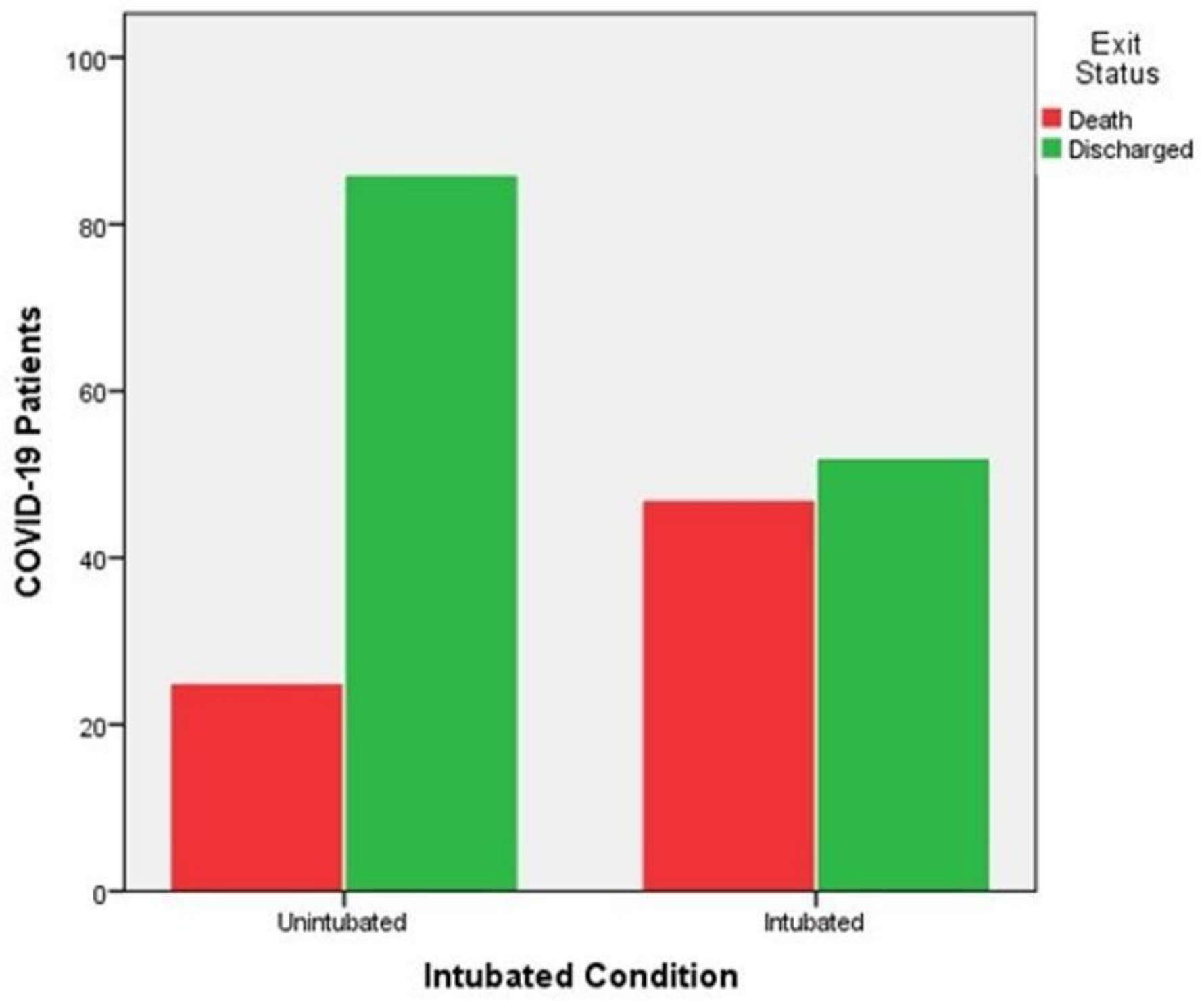

Figure 2

Exit status in COVID-19 patients treated with MSCs according to intubation status. 


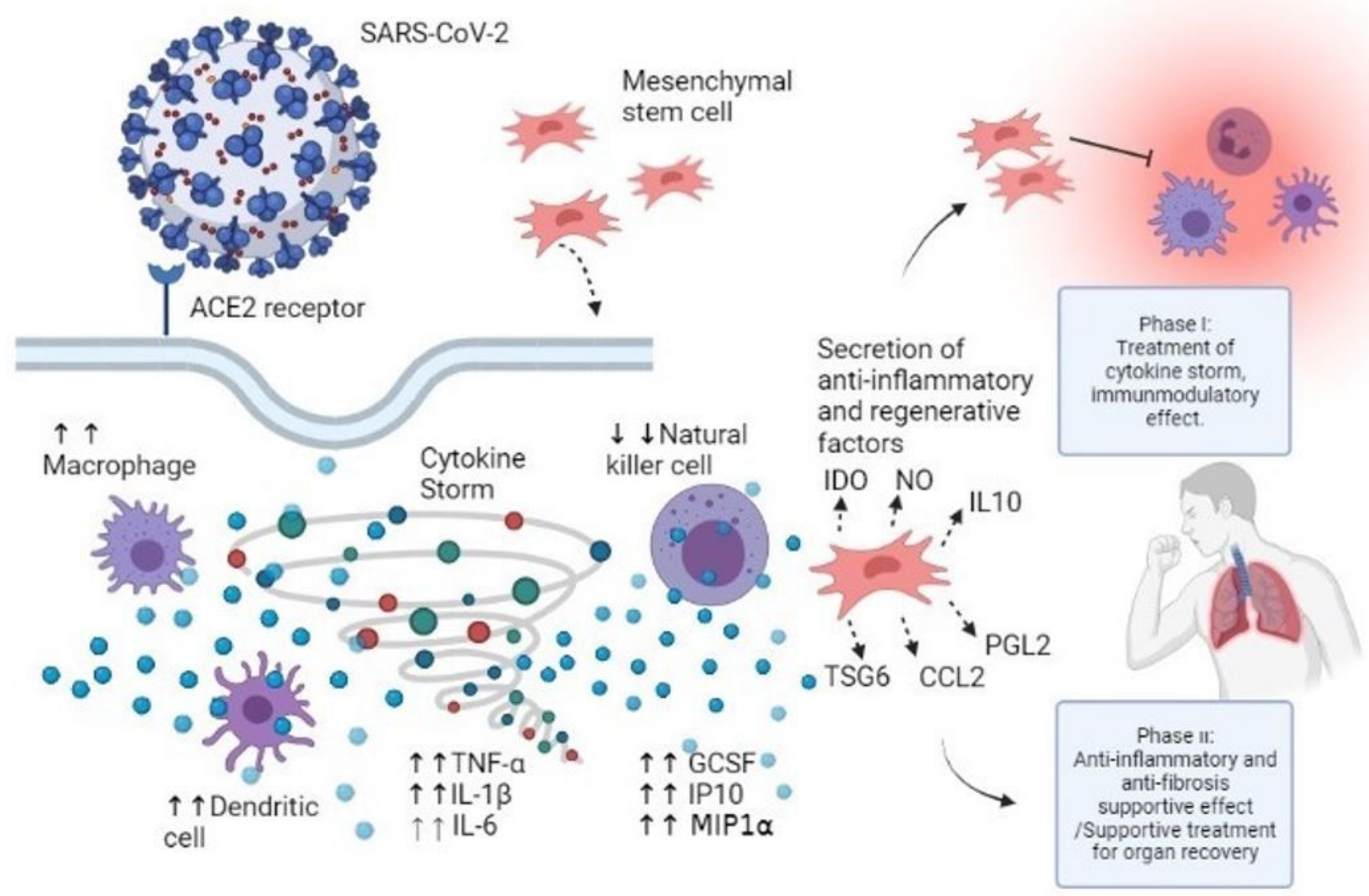

Figure 3

MSCs transplantation improves the damaging effects of the cytokine storm through immunomodulation and improving tissue and organ repair. 